\title{
Redox balance is key to explaining full vs. partial switching to low-yield metabolism
}

Milan JA van Hoek ${ }^{1,2,3}$ and Roeland MH Merks $1,2,3,4^{*}$

\begin{abstract}
Background: Low-yield metabolism is a puzzling phenomenon in many unicellular and multicellular organisms. In abundance of glucose, many cells use a highly wasteful fermentation pathway despite the availability of a highyield pathway, producing many ATP molecules per glucose, e.g., oxidative phosphorylation. Some of these organisms, including the lactic acid bacterium Lactococcus lactis, downregulate their high-yield pathway in favor of the low-yield pathway. Other organisms, including Escherichia coli do not reduce the flux through the high-yield pathway, employing the low-yield pathway in parallel with a fully active high-yield pathway. For what reasons do some species use the high-yield and low-yield pathways concurrently and what makes others downregulate the high-yield pathway? A classic rationale for metabolic fermentation is overflow metabolism. Because the throughput of metabolic pathways is limited, influx of glucose exceeding the pathway's throughput capacity is thought to be redirected into an alternative, low-yield pathway. This overflow metabolism rationale suggests that cells would only use fermentation once the high-yield pathway runs at maximum rate, but it cannot explain why cells would decrease the flux through the high-yield pathway.
\end{abstract}

Results: Using flux balance analysis with molecular crowding (FBAwMC), a recent extension to flux balance analysis (FBA) that assumes that the total flux through the metabolic network is limited, we investigate the differences between Saccharomyces cerevisiae and L. lactis that downregulate the high-yield pathway at increasing glucose concentrations, and E. coli, which keeps the high-yield pathway functioning at maximal rate. FBAwMC correctly predicts the metabolic switching mode in these three organisms, suggesting that metabolic network architecture is responsible for differences in metabolic switching mode. Based on our analysis, we expect gradual, "overflow-like" switching behavior in organisms that have an additional energy-yielding pathway that does not consume NADH ( $e$. g., acetate production in E. coli). Flux decrease through the high-yield pathway is expected in organisms in which the high-yield and low-yield pathways compete for NADH. In support of this analysis, a simplified model of metabolic switching suggests that the extra energy generated during acetate production produces an additional optimal growth mode that smoothens the metabolic switch in E. coli.

Conclusions: Maintaining redox balance is key to explaining why some microbes decrease the flux through the high-yield pathway, while other microbes use "overflow-like" low-yield metabolism.

Keywords: Metabolic switching, Genome-scale metabolic model, Flux Balance Analysis with Molecular Crowding, Overflow metabolism, Redox balance, Escherichia coli, Lactococcus lactis, Saccharomyces cerevisiae

\section{Background}

One of the key steps in energy metabolism is to transfer the energy carried by sugars, including glucose, to the biological "energy currency" adenosine triphosphate (ATP). The number of ATP molecules generated by

\footnotetext{
* Correspondence: Roeland.Merks@cwi.nl

${ }^{1}$ Centrum Wiskunde \& Informatica, Life Sciences, Science Park 123, 1098 XG Amsterdam, The Netherlands

Full list of author information is available at the end of the article
}

metabolizing one molecule of glucose-the ATP yieldis one of the most basic measures of an organism's energy efficiency. One would perhaps expect that evolution has selected organisms for the ability to extract energy from their food at optimal efficiency by maximizing ATP yield. Yet surprisingly, many organisms switch between a high-yield pathway, e.g., aerobic respiration that yields more than thirty moles of ATP per mole glucose, and a highly inefficient, low-yield fermentation

\section{(Ciomed Central}


pathway that yields only two or three moles ATP per mole of glucose. This effect is known as the Crabtreeeffect in the baker's yeast Saccharomyces cerevisiae. S. cerevisiae turns glucose into $\mathrm{CO}_{2}$ in aerobic, glucoselimited conditions. But in abundance of glucose, glucose is converted into ethanol [1], even if oxygen levels do not limit aerobic metabolism. Many bacteria also use a high-yield metabolic pathway in glucose-limited conditions and a low-yield pathway in excess of glucose. Examples are Escherichia coli [2], Bacillus subtilis [3] and lactic acid bacteria, e.g., Lactobacillus plantarum and Lactococcus lactis [4,5]. The effect is also found in multicellular eukaryotes, including human cancer cells, where it is called the Warburg effect [6]. Muscle cells switch to low-yield metabolism during heavy exercise [7], fermenting glucose into lactic acid. Why cells would produce less ATP per glucose molecule than they can is a long-standing question in biology [8-12].

Microbial species show remarkable differences in their metabolic switching strategies. At low glucose concentrations and low growth rates, E. coli uses high-yield metabolism, aerobically converting glucose into $\mathrm{CO}_{2}$ and water. At higher glucose concentrations and fast growth rates, it redirects part of the glucose influx into a low-yield fermentation pathway, keeping oxidative phosphorylation fully active [2]. S. cerevisiae uses highyield, aerobic respiration at slow growth rates; at fast growth rates it ferments most glucose into ethanol, and downregulates aerobic respiration, keeping aerobic respiration active at a much lower rate. Although $L$. lactis does not have an aerobic respiration pathway, it still performs a metabolic switch. At fast growth rates it makes a full switch to lactic acid fermentation [4], which yields about 50\% less ATP than the higher-yield mixed acid fermentation pathway, that produces formate, acetate and ethanol.

A plausible explanation for metabolic switching is "overflow metabolism". It assumes that organisms only switch to low-yield metabolism if the high-yield pathway is operating at maximum rate and cannot process any more molecules $[13,14]$. The remainder would then spill into the low-yield pathway. This explanation requires the low-yield pathway to operate at a faster rate than the high-yield pathway, which is likely the case $[8,15,16]$. Thus overflow metabolism plausibly explains concurrent use of high-yield and low-yield pathways, as in E. coli. However, a problem with overflow metabolism is that it does not explain why organisms like $S$. cerevisiae or $L$. lactis would partly switch off their high-yield pathways at high growth rates.

Recent studies have suggested that the limited amount of metabolic enzymes fitting inside the cell may be key to low-yield metabolism $[12,17,18]$. Simply because cells can host only a finite number of metabolic enzymes, they may need to trade off investment into the bulky enzymatic machinery required for low-throughput, highyield metabolism, or alternatively to invest into many more "lean" glycolytic enzymes producing a highthroughput, low yield metabolism. Thus, according to this view, high glucose uptake rate should correlate with low yield metabolism, and vice versa. Indeed, this is observed in comparative studies of metabolism in yeast species of the Saccharomyces clade [19] and in comparative studies of glucose metabolism of various bacterial species [20].

If cells need to trade off fast metabolism and highyield metabolism, then why do we still observe overflow metabolism, as in E. coli? We address this question by comparing the optimal metabolic switching strategies of L. lactis, S. cerevisiae, and E. coli as predicted by a genome-scale computational model. These three organisms use different pathways to metabolize glucose. In Figure 1 a simplified reaction scheme of the most important glucose degrading pathways in these three organisms is presented. E. coli can use oxidative phosphorylation, lactate fermentation, ethanol fermentation and acetate fermentation. L. lactis can use mixed-acid fermentation, producing formate, acetate and ethanol, or lactate fermentation. S. cerevisiae can use oxidative phosphorylation, ethanol fermentation and acetate fermentation.

To predict the metabolic switches these three organisms can perform, we make use of a variant of Flux Balance Analysis (FBA), a method that calculates fluxes through metabolic networks given constraints on the network and given an objective function to maximize. By maximizing growth rate, FBA often correctly predicts cellular metabolism, including uptake, excretion and growth rates of cells $[2,21]$. However, because the glucose uptake rate is fixed in these simulations, growth yield (defined as the growth rate divided by the glucose uptake rate) is effectively maximized [16]. Therefore, FBA cannot satisfactorily predict low-yield metabolism.

For this reason, we use an extension of FBA, Flux Balance Analysis with Molecular Crowding (FBAwMC) $[17,22]$. In contrast to FBA, FBAwMC calculates the optimal flux distribution through a metabolic network under the physiologically-plausible constraint that only a finite number of metabolic enzymes fit into a cell. Because each of the enzymes has a maximum turnover number $\left(k_{c a t}\right)$, molecular crowding naturally results in a constraint on the total metabolic flux through the network:

$$
\sum c_{i} f_{i} \leq V_{\text {prot }}
$$

with $f_{i}$ being the flux through reaction $i, V_{\text {prot }}$ the volume fraction of macromolecules devoted to metabolic enzymes and $c_{i}$ the "crowding coefficient" of 


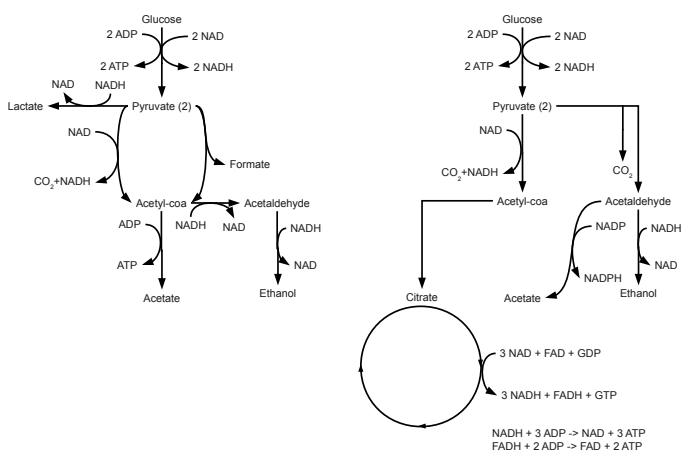

A

B

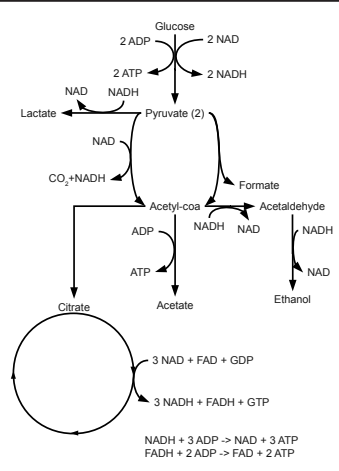

C

Figure 1 Simplified reaction scheme for the 3 organisms studied. A. L. lactis; B. S. cerevisiae; C. E. coli.

reaction $i$. The crowding coefficient of reaction $i$ is defined as the volume that needs to be occupied with enzymes to reach unit flux through reaction $i$ and is given by $c_{i} \equiv \frac{M v_{i}}{V b_{i}}$, where $M$ is the cell mass, $V$ the cell volume, $v_{i}$ the molar volume of the enzyme catalyzing reaction $i$, and $b_{i}$ a variable describing the proportionality between enzyme concentration and flux through reaction $i$ [22]. Intuitively, the crowding coefficient can be seen as the protein cost of a reaction: enzymes with low crowding coefficients have small molecular volume or catalyse fast reactions. FBAwMC correctly predicts low-yield metabolism: e.g., growth curves of E. coli $[17,22]$, and the Warburg effect in cancer cells [18]. Therefore, FBAwMC is well suited for our aim: to unravel the metabolic differences between microbes that decrease the flux through the high-yield pathway at high growth rates and those that keep the high-yield pathway always fully active.

Because crowding coefficients for most metabolic enzymes are unknown, previous studies proposed a range of strategies to estimate them. Beg et al. [22] fitted an average crowding coefficient $\langle c\rangle$ in order to obtain a good match between predicted and measured growth rates. Shlomi et al. [23] obtained 15\% of crowding coefficients from experimental data and assigned the median of the known crowding coefficient values to the remaining unknown crowding coefficients. Vazquez et al. [17] sampled crowding coefficients randomly from a range of physiologically-plausible values obtained from on-line, biochemical databases, and presented averages and variations of the metabolic fluxes predicted for a large random sample of crowding coefficients.

Although the study of an estimated, specific set of crowding coefficients or an average can provide some insight, in reality metabolic networks may operate under an entirely different set of crowding coefficients. Therefore, in the absence of accurate, experimental estimates of crowding coefficients, FBAwMC cannot decide on one real situation. Studying growth yield predictions for large samples of biochemically-plausible sets of crowding coefficients can give more robust insights into the metabolic network than studies with single crowding coefficient estimates, because it reveals what growth yields are most plausible and what are the alternative behaviors of the network.

Our analysis suggests that mechanisms to maintain $\mathrm{NAD}^{+} / \mathrm{NADH}$ ratio are key to the metabolic differences between the two types of metabolic switches. Organisms in which both the high-yield and low-yield pathways reduce $\mathrm{NADH}$ may downregulate high-yield metabolism at high growth rates. If organisms have an additional energy-yielding pathway that does not consume NADH (e.g., acetate production in E. coli), it is optimal to keep both the low-yield and high-yield pathways active at high growth rates.

\section{Results}

\section{Predicted yield distributions reflect metabolic switching strategy}

Using genome-scale stoichiometric networks of L. lactis [24], E. coli [25], and S. cerevisiae [26] we first confirmed that our implementation of the FBAwMC method reproduces the correct growth curves (Figure 2 ). Indeed, FBAwMC qualitatively reproduces both the metabolic switch of $S$. cerevisiae and L. lactis and the overflow metabolism of E. coli. Following Vazquez and coworkers [17], crowding coefficients were chosen at random from a distribution of crowding coefficients based on published molar volumes (Metacyc [27]) and turnover numbers (Brenda [28]). Thus the growth curves that FBAwMC predicts are the average behavior for 1000 randomly sampled sets of crowding coefficients. Figure 2 reports the average and standard deviations of waste product formation or oxygen consumption for the set of simulations resulting in lowyield metabolism (growth yield $<0.3$ gr dry weight/gr 

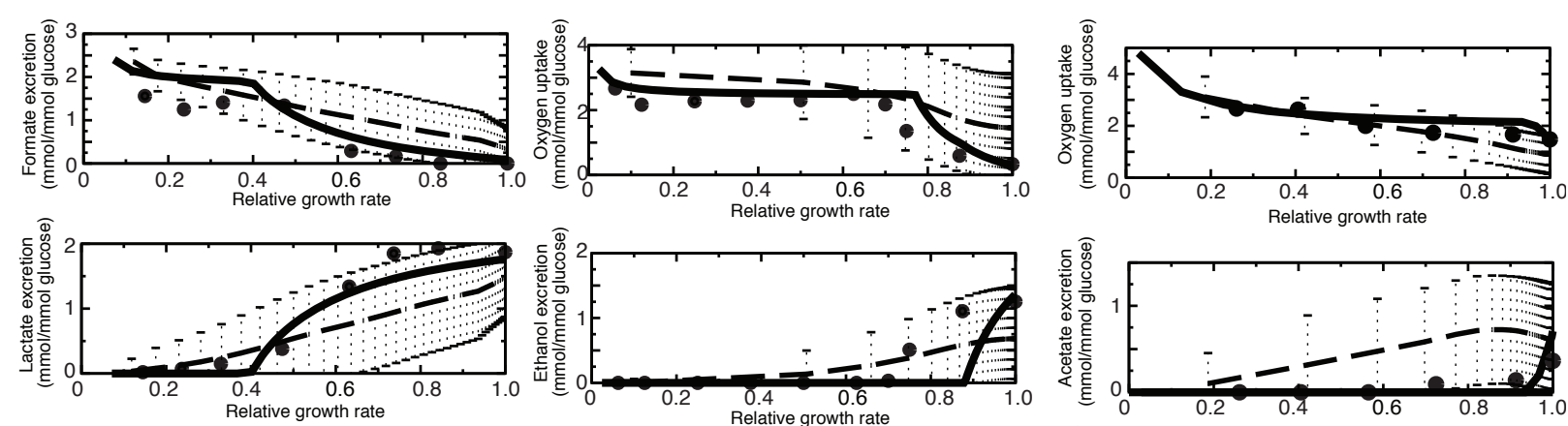

B

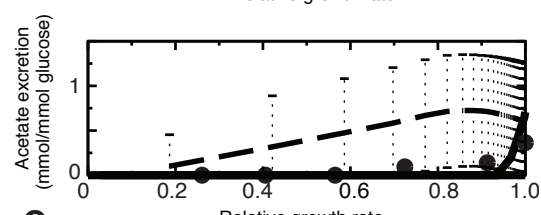

C

Figure 2 FBAwMC growth simulations, compared with experimental data (discs). The best fitting simulation is indicated with a solid line, the mean and standard deviations with dashed lines. Experimental data are indicated with black dots. We scaled the growth rate of the simulations and the experimental observations to the maximal growth rate. Mean and standard deviations are calculated from all simulations that switch to low-yield metabolism at high growth rates (yield < $0.3 \mathrm{gr}$ dry weight/gr glucose). A. L. lactis, data from Thomas et al. [4]; B. S. cerevisiae, data from Hoek et al. [29]; C. E. coli, data from Varma and Palsson [2].

glucose). This represents $74 \%$ of all simulations for $E$. coli, $44 \%$ for L. lactis and $32 \%$ for S. cerevisiae. As reflected by the growth curves' large variation, the optimal growth curve that FBAwMC predicts depends strongly on the particular sample of crowding coefficients used. Because a crowding coefficient $c_{i}$ reflects the enzymatic cost of producing a metabolic flux $f_{i}$, we asked if the relation between crowding coefficient selections and the predicted optimal fluxes could yield new biological insight.

Figure 3 shows the predicted, optimal growth yields at unconstrained glucose influx for a sample of 1000 sets of randomly selected crowding coefficients. Interestingly, computationally obtained growth yields in L. lactis and S. cerevisiae distribute bimodally, with only few simulations predicting intermediate growth yields. By contrast, the predicted growth yields of $E$. coli are distributed more uniformly. Strikingly, the predicted growth yield distributions for a random sampling of crowding coefficients correlate well with the organisms' switching strategies: L. lactis and S. cerevisiae, which downregulate their high-yield metabolism, display a bimodal distribution of predicted growth yields, whereas E. coli, which has overflow-like metabolism, displays a uniform distribution of predicted growth yields.

The distribution of growth yields only gives information of the metabolic behavior at maximal growth rates. Next we tested if individual simulations show overflowlike metabolism or not. Figure 4 shows a frequency diagram of the reduction of the flux through the high-yield pathway at maximal growth rate, as a percentage of the maximal flux through the high-yield pathway. For E. coli we observe that most sets of crowding coefficients lead to little flux decrease through the high-yield pathway (Figure 4C). However, for L. lactis, most sets of crowding coefficients produce a complete halt of the highyield pathway (Figure 4A). For S. cerevisiae, for $40 \%$ of crowding coefficients the high-yield pathway is repressed more than two-fold at high growth rates, whereas for $42 \%$ the high-yield pathway is reduced at most by $10 \%$ (Figure 4B). Thus, at high growth rates FBAwMC predicts overflow-like metabolism in E. coli, whereas it predicts that the flux through the high-yield pathway is likely to be downregulated for L. lactis and S. cerevisiae.

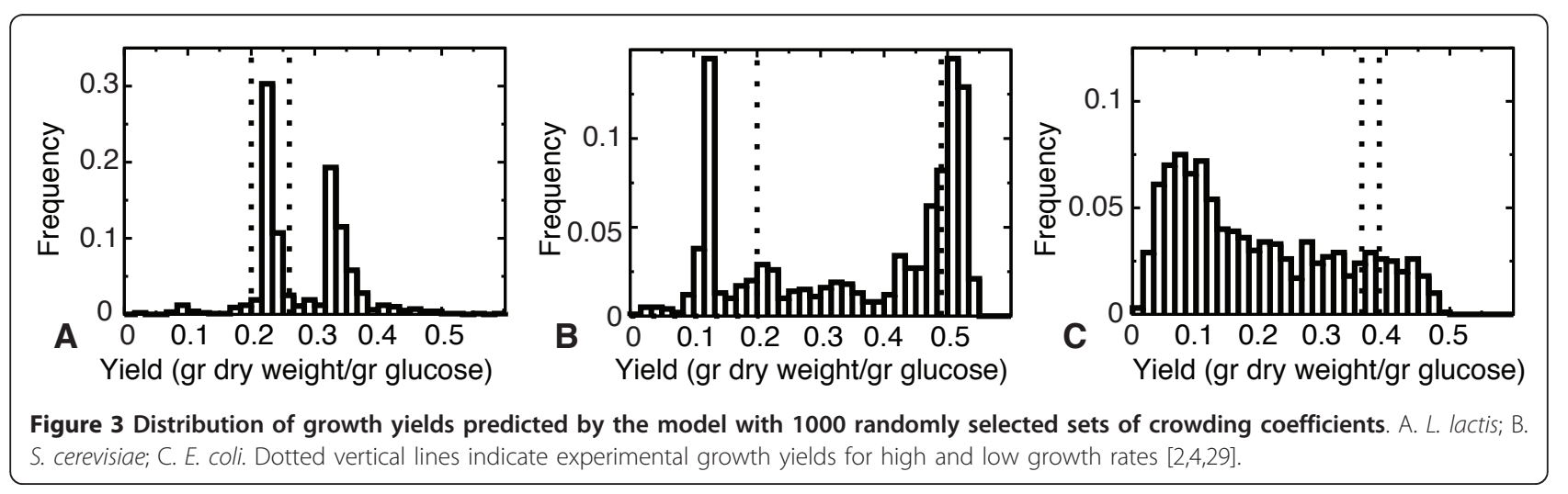



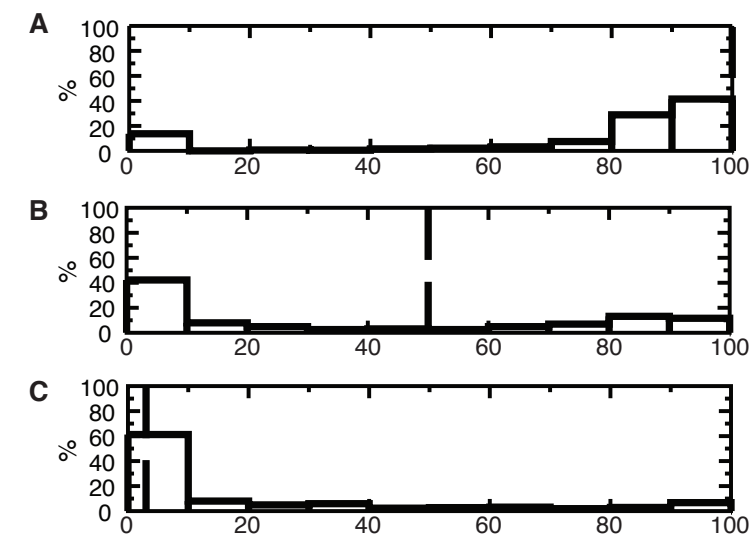

Figure 4 Flux decrease through the high-yield pathway, relative to the maximum flux through the high-yield pathway. This is a measure of the decrease in flux through the high-yield pathway during the metabolic switch. As in Figure 2, we only report simulations that resulted in low-yield metabolism, with yield $<0.3$ gr dry weight/gr glucose. Dashed lines indicate experimental values. A. L. lactis, reported is decrease in formate production rate, data from Thomas et al. [4]; B. S. cerevisiae, reported is decrease in oxygen uptake rate, data from Hoek et al. [29]; C. E. coli, reported is decrease in oxygen uptake rate, data from Varma and Palsson [2].

\section{Acetate excretion makes E. coli use overflow-like switching}

What could explain that S. cerevisiae and L. lactis downregulate high-yield metabolism at high growth rates, whereas E. coli uses overflow-like metabolism? Because we sampled from sets of prokaryotic crowding coefficients for E. coli and L. lactis, and from a eukaryotic dataset for $S$. cerevisiae, we first checked if the speciesspecific sets of crowding coefficients were responsible for our observations. We performed simulations with the $E$. coli network using the eukaryotic set of crowding coefficients and vice versa, and found that this did not affect our results. Therefore we conclude that the key difference between the three models is in the speciesspecific topology of the metabolic networks and their behavior in the presence of crowding, not in the specific values of crowding coefficients.

A key difference that sets $E$. coli apart from L. lactis and $S$. cerevisiae is shown in Figure 1. After converting glucose to pyruvate, L. lactis and S. cerevisiae either convert it into a waste product (ethanol) or further metabolize pyruvate to yield extra ATP. L. lactis converts two acetyl-coA into acetate and ethanol in parallel to retain redox balance, gaining (at most) one additional ATP per mole glucose. S. cerevisiae feeds pyruvate into the citric acid cycle and oxydative phosphorylation, gaining (at most) twenty-eight additional ATP and two GTP per mole glucose. Interestingly, E. coli has three choices: it can convert pyruvate into the waste product ethanol, it can metabolize pyruvate in the citric acid cycle, or it can gain one extra ATP in the conversion of acetyl-coA into acetate.

Although acetate production is a "cheap" way-in terms of the number of enzymes required-to produce additional ATP from pyruvate, it poses an additional challenge to E. coli. During formation of waste products (i.e., lactate or ethanol) the NADH produced in glycolysis or in the conversion from pyruvate to acetyl-coA is reduced back to $\mathrm{NAD}^{+}$. Thus such waste product formation is a "fast" way to restore a sufficiently high NAD +/NADH-ratio. Acetate production does not restore the $\mathrm{NAD}^{+} / \mathrm{NADH}$-ratio, so acetate production might deplete the available $\mathrm{NAD}^{+}$in the cell. So, E. coli might keep oxidative phosphorylation running at fast growth rates (and consume oxygen) in order to profit from the extra ATP yield in acetate formation and restore the NAD + NADH ratio.

This analysis suggests that, if $E$. coli ferments glucose into lactate or ethanol, its oxygen consumption will be reduced. We therefore studied the excretion patterns belonging to different sets of crowding coefficients more carefully. We found that, if a set of crowding coefficients results in acetate fermentation, without any ethanol or lactate fermentation, the cells continue to consume oxygen consumption during the switch. If a set of crowding coefficients results in lactate or ethanol fermentation, the consumption of oxygen is often reduced (Figure 5). Of the fraction of simulations of $E$. coli in which oxygen consumption is reduced during the metabolic switch (see Figure 4C) most produce ethanol and lactate, not acetate (data not shown). Thus, together these simulations are in agreement with the fact that $E$. coli needs to consume oxygen as an external electron acceptor during acetate fermentation in order to maintain a sufficiently high $\mathrm{NAD}^{+} / \mathrm{NADH}$ ratio [30]. If $E$. coli is genetically engineered to eliminate the TCA cycle and all NADHreducing fermentation pathways (i.e., the strain cannot produce lactate or ethanol), it needs dissolved oxygen to grow [30].

To further confirm the hypothesis that at high growth rates overflow metabolism is optimal in E. coli due to acetate excretion, we blocked acetate excretion in the FBAwMC model of $E$. coli (by setting the maximum efflux to zero) such that acetate production stalled, and recalculated the distribution of optimal growth yields. As Figure 6 demonstrates, in this simulation experiment the growth yields become bimodally distributed over the crowding coefficient samples, suggesting that E. coli can switch bimodally. Also, there are more sets of crowding coefficients that result in a decrease in flux through the high-yield pathway (Additional file 1: Figure S1). Apparently, after blocking the route for producing one extra ATP, it again becomes optimal to restore the NAD 

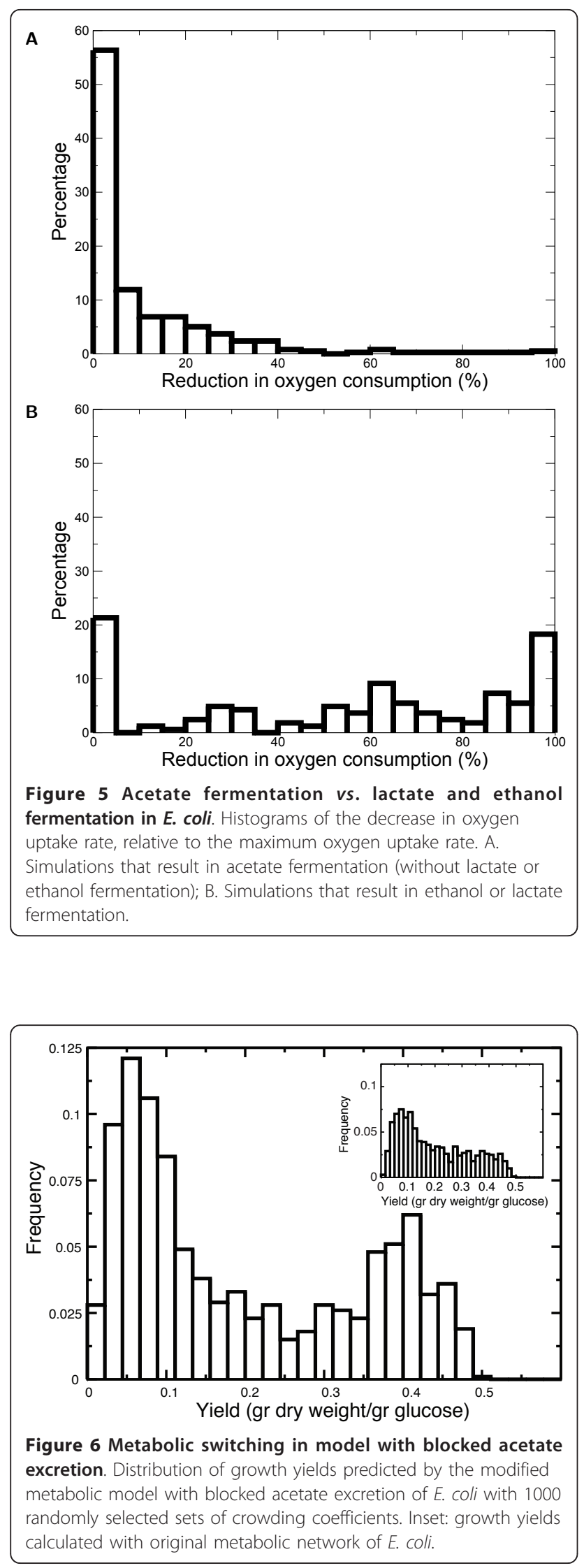

+/NADH ratio by producing the alternative waste products lactate or ethanol. This model observation agrees with experiments by De Mey et al. [31] who report increased lactate and ethanol excretion after reducing the carbon flow to acetate.

To confirm that an additional ATP-producing pathway can indeed lead to an additional optimal growth mode, we developed a simplified metabolic network model [16], illustrated in Figure 7A. The simplified model has five reactions that represent glycolysis, lactate/ethanol excretion, acetate excretion, the TCA-cycle, and oxidative phosphorylation. Using FBAwMC we predicted the optimal yields for a sample of crowding coefficients. In this model, we found four metabolic modes (Figure 7B). After knocking out the acetate pathway, we found only two metabolic modes, a high-yield and a low-yield pathway (Figure 7C). Thus, also in this simplified model, acetate production introduces intermediateyield metabolic modes.

\section{Discussion}

We have computationally compared metabolic switching at high growth rates in E. coli with L. lactis and S. cerevisiae. E. coli shows overflow metabolism, meaning that at high growth rates it increases its metabolic rate by activating low-yield metabolic pathways in addition to the high-yield oxidative phosphorylation pathway. Instead, L. lactis and S. cerevisiae show metabolic switching: they suppress the flux through their highyield pathways at high growth rates, relying mostly on low-yield metabolism. Our analysis suggests that a key difference between the two groups is the number of metabolic pathways yielding ATP, and the effect of these pathways on the $\mathrm{NAD}^{+} / \mathrm{NADH}$-ratio. L. lactis and

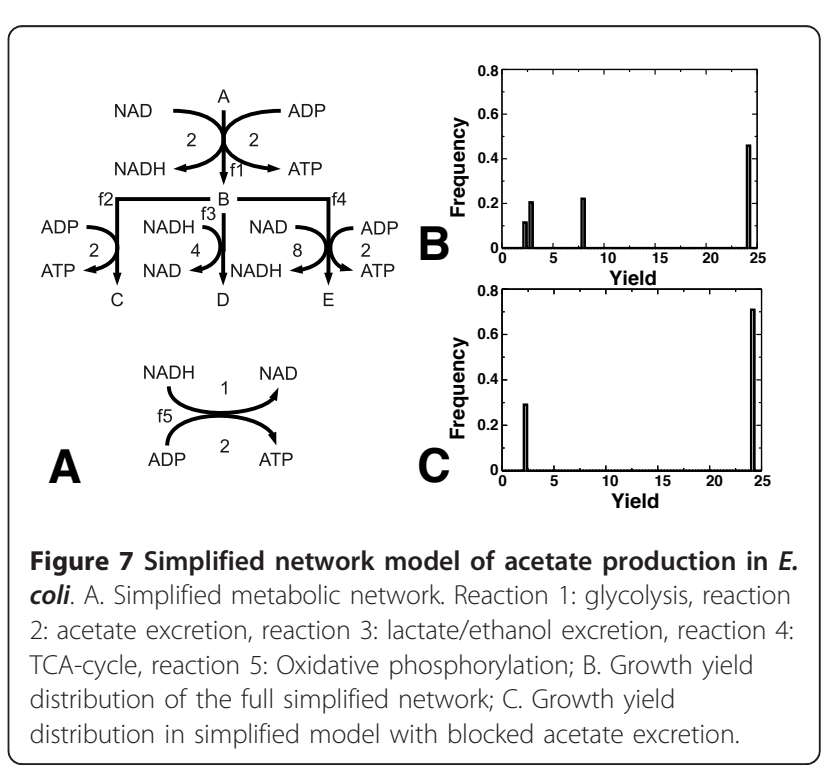


S. cerevisiae have two alternative pathways, an efficient, high-yield pathway-glycolysis followed by mixed acid fermentation or oxidative phosphorylation-and an inefficient, low-yield pathway-glycolysis followed by lactic acid or ethanol production. In both the low-yield and high-yield pathways, the NADH resulting from glycolysis is oxidized back to $\mathrm{NAD}^{+}$. In addition to lactate and ethanol fermentation and oxidative phosphorylation, $E$. coli has a second low-yield pathway: the conversion of pyruvate to acetate. This pathway yields one extra ATP over lactate fermentation, but it does not oxidize $\mathrm{NADH}$, so the $\mathrm{NAD}^{+} / \mathrm{NADH}$-ratio must be restored elsewhere. Our model suggests that in this case it is optimal to keep oxidative phosphorylation running, instead of calling in low-yield pathways to reduce $\mathrm{NADH}$, e.g., lactate production.

To test the idea that acetate production is the cause of overflow metabolism in E. coli, we blocked acetate fermentation in the FBAwMC model. The distribution of predicted growth yields became more bimodal, and the proportion of cells that downregulated their high-yield pathway increased. Should we hence expect E. coli to downregulate its high-yield pathway at high growth rates, if its acetate production pathway were blocked experimentally? Note that FBAwMC predicts optimal growth rates. Thus it predicts the growth rates for organisms that have already evolved towards optimality. Our results would therefore suggest that mutated E. coli strain with blocked acetate fermentation would evolve downregulation of its high-yield pathway after selection for growth rate in cell culture experiments.

Our model results suggest that restoring the redox balance is key in metabolic switching, agrees with experimental observation. Vemuri et al. [32] overexpressed both NADH oxidase (NOX) and alternative oxidase (AOX) in $S$. cerevisiae and found that glycerol (for NOX) or ethanol formation (for AOX) were diminished. In another study, Vemuri et al. [33] increased oxidation of NADH by overexpressing NOX in E. coli and studied the effect on overflow metabolism. They found that overexpression of NOX strongly diminished acetate fermentation.

To check whether our model is consistent with these experiments, we mimicked them in the FBAwMC models for $E$. coli and $S$. cerevisiae. We introduced the reactions that NOX and AOX catalyze to the metabolic model and enforced a lower bound on their fluxes to mimic the effect of overexpression. We performed simulations of S. cerevisiae with ten thousand sets of crowding coefficients, of which only 110 crowding coefficient selections resulted in excretion of both ethanol and glycerol. We went on with these sets of crowding coefficients, because they best mimicked the wild-type phenotype that Vemuri et al. [33] used. The simulated overexpression of NOX or AOX reduced excretion of ethanol and glycerol in practically all of these 110 simulations (Table 1). Thus, in agreement with experiments, the FBAwMC model of $S$. cerevisiae suggests that excessive NADH breakdown reduces ethanol and glycerol fermentation. In the FBAwMC model of E. coli we found that increased oxidation of NADH could either result in a decreased or in an increased production of acetate, depending on the selection of crowding coefficients. Thus, the experiments of Vemuri et al. [33] do not corroborate nor falsify our model. The reason is that FBAwMC identifies optimal fluxes. In E. coli, during acetate fermentation some NADH is formed in the conversion of pyruvate to acetyl-coA. During NOX overexpression, the cells must boost $\mathrm{NADH}$ production to maintain optimal growth; this can be done either using the TCA-cycle or using acetate fermentation. Depending on the crowding coefficients, either way is optimal.

The computational results presented in this paper are contingent on two underlying, biological assumptions of FBAwMC that may limit the applicability of our approach to strains growing in well-mixed, nutrient-rich lab conditions: a) evolution optimizes cells' growth rates instead of yields, and b) a solvent constraint (i.e., the number of enzymes "fitting" inside the cell) puts selective pressure on cells to evolve mechanisms to rapidly produce or remove enzymes for alternative metabolic pathways [22]. Thus FBAwMC implicitly assumes that evolution has shaped cells to make the optimal choice between alternative metabolic pathways.

The optimality assumption is not necessarily correct in all environments. Apart from the fact that evolution does not always lead to optimality [34], game theory suggests that spatial or seasonal environments favor maximization of growth yield $[35,36]$. Optimization of growth rate, as implicitly assumed in FBAwMC, is more likely applicable to homogeneous, non-seasonal environments, i.e., a chemostat $[35,37]$. Thus our simulations apply primarily to laboratory strains, which are adapted to well-mixed, nutrient-rich laboratory conditions. For natural strains, the maximization of growth yield that standard FBA assumes might be better applicable [20].

The second key assumption of FBAwMC, namely that cells have evolved regulation mechanisms to activate production of enzymatic machinery for the pathway

Table 1 Effect of NOX or AOX overexpression on lowyield metabolism

\begin{tabular}{lll}
\hline & NOX & AOX \\
\hline S. cerevisiae ethanol & $96 \%$ & $98 \%$ \\
S. cerevisiae glycerol & $100 \%$ & $97 \%$ \\
E. coli acetate & $16 \%$ & \\
\hline
\end{tabular}

We report the percentage of simulations that result in a decrease in acetate fermentation (for $E$. coli) or ethanol and glycerol fermentation (for S. cerevisiae) 
giving optimal growth rate [22], relates closely to the explanation proposed by Molenaar et al. [12]. Using a minimal model of a self-replicator, they showed how a trade-off between the metabolic efficiency of a pathway, and the cost associated with producing the enzymes for that payway, can lead to a switch in metabolic strategy. Very recently, Zhuang et al. [38] proposed instead that competition for membrane space between glucose transporters and respiratory chain enzymes could be responsible for the metabolic switch between respirative and respiro-fermentative metabolism in E. coli. They introduced an alternative extension of FBA to accommodate for this effect. We anticipate that our results would hold if we used Zhuang et al.'s modification of FBA instead of FBAwMC. A requirement for our results is that the sum of a set of key metabolic fluxes is constrained. In FBAwMC this constraint is proposed to be due to the limited enzyme solvent capacity in the cytosol $[12,17]$. Mathematically, Zhuang et al. [38] propose a very similar constraint, but argue it is due to competition for membrane space between glucose enzymes and respiratory chain enzymes. In fact, the explanation proposed by Vazquez et al. [17] may be more generally applicable, because Zhuang et al.'s rationale would not hold if glucose transporters and respiratory chain enzymes do not share the same membranes as, e.g., in eukaryotes.

\section{Conclusions}

Why, at high rates, do some microbes use low-yield metabolism in addition to the high-yield pathway-overflow metabolism-whereas other microbes downregulate their high-yield pathways? Here we show that maintaining redox balance is key to understanding overflow metabolism in E. coli. Microbes that use low-yield pathways converting NADH back to NAD, including $L$. lactis and $S$. cerevisiae, are expected to downregulate their high-yield pathways at high growth rates. E. coli can get one extra ATP using acetate secretion; doing so it must keep the oxidative phosphorylation pathway running to restore redox balance, giving rise to "overflow-like" metabolism.

\section{Methods}

\section{Flux balance analysis with molecular crowding}

We have used FBAwMC [17,22] to predict growth, uptake and excretion rates in S. cerevisiae, E. coli and $L$. lactis, using the genome-scale metabolic models published in [26,25] and [24], respectively. We downloaded the $E$. coli and $S$. cerevisiae model from the BiGG database [39] http://bigg.ucsd.edu/. The L. lactis model was downloaded from the Supplementary Materials in Oliveira et al. [24].

FBAwMC assumes that the metabolic network is in steady state

$$
\frac{d \vec{x}}{d t}=S \cdot \vec{f}=0
$$

where $\vec{x}$ is a vector of all metabolites, $\vec{f}$ is a vector describing the metabolic flux through each reaction in the network, and $S$ the stoichiometric matrix. $S$ is defined as follows: if reaction $i$ produces $n$ metabolites of type $j$, then $S_{i j} \equiv n ; S_{i j} \equiv-n$ for consumption of metabolite $j$; otherwise $S_{i j} \equiv 0$. FBAwMC attempts to find a solution F of Eq. 2 that maximizes an objective function, given a set of constraints. In this study, we always optimize for growth rate. We also incorporate constraints on the individual fluxes:

$$
f_{l b, n} \leq \mathrm{F}_{n} \leq f_{u b, n}
$$

where $f_{l b, n}$ is the minimal flux and $f_{u b, n}$ the maximal flux through reaction $n$. Furthermore, a constraint on the total flux through the network is added to account for the limited amount of enzymes in any given cell, given by

$$
\sum c_{n} f_{n} \leq V_{\text {prot }}
$$

Here $c_{n} \equiv \frac{M v_{n}}{V b_{n}}$ is the "crowding coefficient", $M$ the cell mass, $V$ the cell volume, $v_{n}$ the molar volume of the enzyme catalysing reaction $n$ and $b_{n}$ a parameter describing the proportionality between enzyme concentration and flux. For a derivation of Eq. 3 see Beg et al. [22]. $V_{\text {prot }}$ is a constant $\left(0 \leq V_{\text {prot }} \leq 1\right)$ representing the volume fraction of macromolecules devoted to metabolic enzymes. We fit $V_{\text {prot }}$ to the experimentally observed growth rate and glucose uptake rate. Additional file 2: Table S1 lists the results of this fitting procedure. Interestingly, $V_{\text {prot }}$ values are very similar across different organisms, ranging from 0.15-0.2. Note that Vazquez et al. [17] assumed that $V_{\text {prot }}=1$, which we believe is unrealistic, because not only metabolic enzymes fill the cell's cytoplasm. Linear programming efficiently solves this problem, but the solution is not necessarily unique.

\section{Crowding coefficients}

To obtain the crowding coefficients $c_{i}$ we adopted the approach of Vazquez et al. [17]. The molar volume $v_{i}$ can be estimated from the molar masses of the enzymes using a specific protein volume of $0.73 \mathrm{ml} / \mathrm{g} . b_{i}$ depends on the concentration of metabolites and on the turnover numbers of the enzymes (for example in a MichaelisMenten way, where $b=V_{\max } \frac{S}{S+K_{M}}$, here, we would estimate $\left.b=V_{\text {max }}\right)$. Following Vazquez et al. [17], we constructed a distribution of crowding coefficients from turnover numbers and enzyme masses. We obtained 
turnover numbers from enzyme database Brenda [28], enzyme masses from MetaCyc [27]. The distribution of crowding coefficients we then obtained using the relationship $c_{i} \equiv \frac{M v_{i}}{V b_{i}}$. The turnover numbers and enzyme masses used are given in Additional file 3: Figure S2.

As there is insufficient data for $L$. lactis we used $E$. coli crowding coefficients for this organism as well. The turnover numbers, both for E. coli and S. cerevisiae varied over orders of magnitudes. Importantly, a few enzyme-substrate combinations had extremely low turnover numbers that effectively stopped the reactions. Because these turnover numbers typically occurred for non-metabolic reactions (e.g., DNA repair) or for nontypical substrates of metabolic enzymes, we only used turnover numbers of metabolic enzymes and for each enzyme we only kept the highest available turnover number and left out enzymes with turnover number smaller than $0.01 / \mathrm{s}$. We used wild-type turnover numbers if reported. The resulting distribution of crowding coefficients for $E$. coli was similar to the distribution found by Vazquez et al. [17] (see Additional file 4: Figure S3). For S. cerevisiae we found a similar distribution as for E. coli (Additional file 5: Figure S4).

\section{In silico growth experiments}

We initiated each simulation with randomly select crowding coefficients from the obtained distributions. We assigned a crowding coefficient of 0 to non-enzymatic reactions. The COBRA Toolbox [40] was used to perform FBAwMC in Matlab, with the GNU Linear Programming Kit as linear programming solver http://www. gnu.org/software/glpk.

The in silico growth media included the vitamins, nucleotides and minerals required for optimal growth. For the constraints on the reactions used in the simulations, we refer to Additional file 6: Table S2. Because L. lactis cannot synthesize many amino acids we must supply them in the in silico growth medium. In order to ensure that cells are not limited by amino acid uptake, we constrained the maximal amino acid uptake rates to the biomass content of that amino acid multiplied by twice the (experimentally observed) maximal growth rate. In this way, the maximal amino acid uptake rate suffices for twice the experimentally observed growth rate.

Matlab code to reproduce the simulations are included in Additional file 7. The COBRA Toolbox [40] and a linear programming solver are required.

\section{Additional material}

Additional file 1: Figure S1. Histogram of decrease in oxygen uptake for E. coli, when acetate excretion is allowed (black) and knocked out (red). When acetate excretion is knocked out, there are more simulations that become fully high-yield, but also more that stop consuming oxygen.
Additional file 2: Table S1. Table describing the summary of fitting $V_{\text {prot }}$ to experimental growth rate and glucose uptake rate. For every organism, we varied $V_{\text {prot }}$ (volume fraction of macromolecules devoted to metabolic enzymes) between 0 and 1 and performed, for each value of $V_{\text {prot, }} 1000$ simulations with random sets of crowding coefficients. For the simulations described in this paper, we used the value of $V_{\text {prot }}$ that

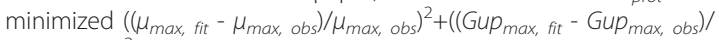
Gup $_{\max }$, obs $)^{2}$. Here, $\mu_{\max }$ fit $\mu_{\max }$ obs are the fitted and observed maximal growth rate and Gupmax, fit Gupmax, obs are the fitter and observed maximal glucose uptake rate. In this table, $P_{\text {ineff }}$ indicates the fraction of the 1000 simulations that exhibits low-yield metabolism, which was defined as having a growth yield $<0.3 \mathrm{gr} / \mathrm{gr}$ glucose. Experimental data is from Hoek et al. [29]; Thomas et al. [4]; Varma and Palsson [2].

Additional file 3: Figure S2. Excel file with turnover numbers and enzyme masses used to calculate the crowding coefficients.

Additional file 4: Figure S3. Histograms of turnover numbers (1/s) (A) and crowding coefficients (gram DW hr/mmol) (B) of E. coli. A. All turnover numbers of E. coli in BRENDA (Chang et al. [28]); B. Crowding coefficients resulting from all turnover numbers of $E$. coli in BRENDA (Chang et al. [28]); C. Turnover numbers of E. coli used for the simulations; D. Crowding coefficients of E. coli used in the simulations; E. Turnover numbers as used in Vazquez et al. [17]; F. Crowding coefficients as used in Vazquez et al. [17].

Additional file 5: Figure S4. Histograms of turnover numbers (1/S) $(A, C)$ and crowding coefficients (gram DW hr/mmol) $(B, D)$ of $S$. cerevisiae. A. All turnover numbers of $S$. cerevisiae in BRENDA (Chang et al. [28]); B.

Crowding coefficients resulting from all turnover numbers of $S$. cerevisiae in BRENDA (Chang et al. [28]); C. Turnover numbers of S. cerevisiae used for the simulations; D. Crowding coefficients of S. cerevisiae used in the simulations.

Additional file 6: Table S2. Excel file describing, for every reaction, the lower and upper bounds used in the simulations.

Additional file 7: Mini-website with Matlab code and instructions for reproducing the simulations.

\section{Abbreviations}

AOX: Alternative oxidase; ATP: Adenosine triphosphate; BiGG: Biochemical Genetic and Genomic knowledgebase of large scale metabolic reconstructions; $\mathrm{CO}_{2}$ : Carbon dioxide; COBRA: COnstraints Based Reconstruction and Analysis; FBA: Flux-balance analysis; FBAwMC: Fluxbalance analysis with molecular crowding; GTP: Guanosine-5'-triphosphate; $\mathrm{NAD}^{+}$: Nicotinamide Adenine Dinucleotide; NADH: Reduced form of $\mathrm{NAD}^{+}$; NOX: NADH oxidase.

\section{Acknowledgements}

We thank three anonymous referees for their constructive comments that have helped us to improve the manuscript. This work was cofinanced by the Netherlands Consortium for Systems Biology (NCSB), which is part of the Netherlands Genomics Initiative/Netherlands Organisation for Scientific Research.

\section{Author details}

${ }^{1}$ Centrum Wiskunde \& Informatica, Life Sciences, Science Park 123, 1098 XG Amsterdam, The Netherlands. ${ }^{2}$ Netherlands Institute for Systems Biology, Science Park 123, 1098 XG Amsterdam, The Netherlands. ${ }^{3}$ Netherlands Consortium for Systems Biology, Amsterdam, The Netherlands. ${ }^{4}$ Mathematical Institute, Leiden University, P.O. Box 9512, 2300 RA Leiden, The Netherlands.

\section{Authors' contributions}

$\mathrm{MvH}$ designed the model and performed the simulations. RM and MvH conceived of the study and drafted the manuscript. All authors read and approved the final manuscript.

\section{Competing interests}

The authors declare that they have no competing interests. 
Received: 13 January 2012 Accepted: 24 March 2012

Published: 24 March 2012

\section{References}

1. van Dijken JP, Weusthuis RA, Pronk JT: Kinetics of growth and sugar consumption in yeasts. Antonie Van Leeuwenhoek 1993, 63(3-4):343-352.

2. Varma $A$, Palsson $B \varnothing$ : Stoichiometric flux balance models quantitatively predict growth and metabolic by-product secretion in wild-type Escherichia coli W3110. Appl Environ Microbiol 1994, 60(10):3724-3731.

3. Dauner M, Storni T, Sauer U: Bacillus subtilis metabolism and energetics in carbon-limited and excess-carbon chemostat culture. J Bacteriol 2001 183(24):7308-7317.

4. Thomas TD, Ellwood DC, Longyear VM: Change from homo- to heterolactic fermentation by Streptococcus lactis resulting from glucose limitation in anaerobic chemostat cultures. J Bacterio/ 1979, 138:109-117.

5. Teusink B, Wiersma A, Molenaar D, Francke C, de Vos WM, Siezen RJ, Smid EJ: Analysis of growth of Lactobacillus plantarum WCFS1 on a complex medium using a genome-scale metabolic model. $J$ Biol Chem 2006, 281(52):40041-40048

6. Kim JW, Dang CV: Cancer's molecular sweet tooth and the Warburg effect. Cancer Res 2006, 66(18):8927-8930.

7. Robergs RA, Ghiasvand F, Parker D: Biochemistry of exercise-induced metabolic acidosis. Am J Physiol Regul Integr Comp Physiol 2004, 287(3) R502-R516.

8. Westerhoff HV, Hellingwerf KJ, Dam KV: Thermodynamic efficiency of microbial growth is low but optimal for maximal growth rate. $P$ Natl Acad Sci USA 1983, 80:305-309.

9. Tempest DW, Neijssel OM: Physiological and energetic aspects of bacterial metabolite overproduction. FEMS Microbiol Lett 1992, 79(13):169-176.

10. Russell JB, Cook GM: Energetics of bacterial growth: balance of anabolic and catabolic reactions. Microbiol Rev 1995, 59:48-62.

11. Russell JB: The energy spilling reactions of bacteria and other organisms. J Mol Microbiol Biotechnol 2007, 13(1-3):1-11

12. Molenaar $D$, van Berlo $R$, de Ridder $D$, Teusink B: Shifts in growth strategies reflect tradeoffs in cellular economics. Mol Syst Biol 2009, 5:323.

13. Andersen KB, Von Meyenburg K: Are growth rates of Escherichia coli in batch cultures limited by respiration? J Bacterio/ 1980, 144:114-123.

14. Holms H: Flux analysis and control of the central metabolic pathways in Escherichia coli. FEMS Microbio/ Rev 1996, 19(2):85-116

15. Heinrich R, Montero F, Klipp E, Waddell TG, Melendez-Hevia E: Theoretical approaches to the evolutionary optimization of glycolysis: thermodynamic and kinetic constraints. Eur J Biochem 1997, 243(12):191-201.

16. Schuster $S$, Pfeiffer $T$, Fell DA: Is maximization of molar yield in metabolic networks favoured by evolution? J Theor Biol 2008, 252(3):497-504

17. Vazquez A, Beg QK, Demenezes MA, Ernst J, Bar-Joseph Z, Barabasi AL, Boros LG, Oltvai ZN: Impact of the solvent capacity constraint on E. coli metabolism. BMC Syst Biol 2008, 2:7.

18. Vazquez A, Liu J, Zhou Y, Oltvai ZN: Catabolic efficiency of aerobic glycolysis: the Warburg effect revisited. BMC Syst Biol 2010, 4:58.

19. Merico A, Sulo P, Piskur J, Compagno C: Fermentative lifestyle in yeasts belonging to the Saccharomyces complex. FEBS J 2007, 274(4):976-989.

20. Fuhrer $T$, Fischer $E$, Sauer U: Experimental identification and quantification of glucose metabolism in seven bacterial species. J Bacteriol 2005 , 187(5):1581-1590.

21. Edwards JS, Palsson BØ: The Escherichia coli MG1655 in silico metabolic genotype: its definition, characteristics, and capabilities. P Natl Acad Sci USA 2000, 97(10):5528-5533.

22. Beg QK, Vazquez A, Ernst J, de Menezes MA, Bar-Joseph Z, Barabasi AL, Oltvai ZN: Intracellular crowding defines the mode and sequence of substrate uptake by Escherichia coli and constrains its metabolic activity. P Natl Acad Sci USA 2007, 104(31):12663-12668.

23. Shlomi T, Benyamini T, Gottlieb E, Sharan R, Ruppin E: Genome-scale metabolic modeling elucidates the role of proliferative adaptation in causing the Warburg effect. PLoS Comput Biol 2011, 7(3):e1002018.

24. Oliveira AP, Nielsen J, Forster J: Modeling Lactococcus lactis using a genome-scale flux model. BMC Microbiol 2005, 5:39.

25. Feist AM, Henry CS, Reed JL, Krummenacker M, Joyce AR, Karp PD, Broadbelt LJ, Hatzimanikatis $\vee$, Palsson $B \varnothing$ : A genome-scale metabolic reconstruction for Escherichia coli K-12 MG1655 that accounts for 1260 ORFs and thermodynamic information. Mol Syst Biol 2007, 3:121.

26. Duarte NC, Herrgard MJ, Palsson $B \varnothing$ : Reconstruction and validation of Saccharomyces cerevisiae iND750, a fully compartmentalized genomescale metabolic model. Genome Res 2004, 14(7):1298-1309.

27. Caspi R, Foerster H, Fulcher CA, Kaipa P, Krummenacker M, Latendresse M, Paley S, Rhee SY, Shearer AG, Tissier C, Walk TC, Zhang P, Karp PD: The MetaCyc Database of metabolic pathways and enzymes and the BioCyc collection of Pathway/Genome Databases. Nucleic Acids Res 2008, 36(Database):D623-D631

28. Chang A, Scheer M, Grote A, Schomburg I, Schomburg D: BRENDA, AMENDA and FRENDA the enzyme information system: new content and tools in 2009. Nucleic Acids Res 2009, 37:(Database issue):D588-D592.

29. Hoek PV, Dijken JPV, Pronk JT: Effect of specific growth rate on fermentative capacity of baker's yeast. Appl Environ Microbiol 1998, 64(11):4226-4233.

30. Causey TB, Zhou S, Shanmugam KT, Ingram LO: Engineering the metabolism of Escherichia coli W3110 for the conversion of sugar to redox-neutral and oxidized products: homoacetate production. P Nat Acad Sci USA 2003, 100(3):825-832.

31. Mey MD, Lequeux GJ, Beauprez JJ, Maertens J, Horen EV, Soetaert WK, Vanrolleghem PA, Vandamme EJ: Comparison of different strategies to reduce acetate formation in Escherichia coli. Biotechnol Progr 2007, 23(5):1053-1063

32. Vemuri GN, Eiteman MA, McEwen JE, Olsson L, Nielsen J: Increasing NADH oxidation reduces overflow metabolism in Saccharomyces cerevisiae. $P$ Natl Acad Sci USA 2007, 104(7):2402-2407.

33. Vemuri GN, Altman E, Sangurdekar DP, Khodursky AB, Eiteman MA: Overflow metabolism in Escherichia coli during steady-state growth: transcriptional regulation and effect of the redox ratio. Appl Environ Microbiol 2006, 72(5):3653-3661.

34. Bull JJ, Wang IN: Optimality models in the age of experimental evolution and genomics. J Evolution Biol 2010, 23(9):1820-1838.

35. Pfeiffer T, Schuster S, Bonhoeffer S: Cooperation and competition in the evolution of ATP-producing pathways. Science 2001, 292(5516):504-507.

36. MacLean RC, Gudelj I: Resource competition and social conflict in experimental populations of yeast. Nature 2006, 441(7092):498-501.

37. Pfeiffer T, Schuster S: Game-theoretical approaches to studying the evolution of biochemical systems. Trends Biochem Sci 2005, 30:20-25.

38. Zhuang K, Vemuri GN, Mahadevan R: Economics of membrane occupancy and respiro-fermentation. Mol Syst Biol 2011, 7:500.

39. Schellenberger J, Park JO, Conrad TM, Palsson BØ: BiGG: a Biochemical Genetic and Genomic knowledgebase of large scale metabolic reconstructions. BMC Bioinformatics 2010, 11:213.

40. Becker SA, Feist AM, Mo ML, Hannum G, Palsson BØ, Herrgard M: Quantitative prediction of cellular metabolism with constraint-based models: the COBRA Toolbox. Nat Protoc 2007, 2(3):727-738.

doi:10.1186/1752-0509-6-22

Cite this article as: van Hoek and Merks: Redox balance is key to explaining full vs. partial switching to low-yield metabolism. $B M C$ Systems Biology 2012 6:22

\section{Submit your next manuscript to BioMed Central and take full advantage of:}

- Convenient online submission

- Thorough peer review

- No space constraints or color figure charges

- Immediate publication on acceptance

- Inclusion in PubMed, CAS, Scopus and Google Scholar

- Research which is freely available for redistribution 